\title{
Epiglottitis in Canada: A multiregional review
}

\author{
BARBARA J LAW, MD, DAVID DRAPER, MD, Elaine L MILLS, MD, MANON ALLARD, MD, CherI NIJSSEN-JORDAN, MD, \\ ROBERT BORTOLIOSSI, MD, NONI E MACDONALD, MD, ABDULAZIZ A AL-TWAIM, MD, WILLIAM ALBRITON, MD, PHD, \\ GORDON KASIAN, MD, LOTIIE REA, RN, SHARON CRONK, RN, ROBERT MORRIS, MD
}

\begin{abstract}
Epiglottitis is an acute, life threatening infection usually caused by Haemophilus influenzae type b. Although antibiotic therapy is an important part of management, the optimal route and duration is unknown. A multicentre retrospective review of 305 children with epiglottitis was carried out in order to relate antibiotic therapy to hospital course and outcome, as well as to examine regional variation in patient demographics, clinical presentation and course of disease. A standardized form was used to extract data from hospital records. Although management varied significantly among the six centres in terms of mean duration of intubation ( 46 to $81 \mathrm{~h}$ ), intravenous antibiotic therapy ( 3.8 to 5.7 days) and hospital stay ( 5.3 to 8.4 days), there were no significant centre-related differences in epidemiology, clinical course or outcome of epiglottitis. An
\end{abstract}

extraepiglottic focus of infection was present in $15 \%$ of patients and included three with septic arthritis and one with meningitis. The duration of fever in hospital and maximum recorded temperature in hospital were significantly greater for children with extraepiglottic infection compared to those with epiglottitis alone. The data presented in this review suggest that most children with epiglottitis have an uncomplicated course and respond rapidly to antimicrobial therapy following airway securement. A short period of intravenous and oral antibiotic therapy is likely adequate for most children with epiglottitis. A well designed multicentre prospective trial is still needed to determine the optimal duration of antibiotic therapy. Can J Infect Dis 1990;1(1):15-22

Key Words: Epiglottitis, Haemophilus influenzae type $b$
$\mathrm{E}_{\mathrm{n}}^{\mathrm{p}}$ PIGLOTTITIS IS AN ACUTE, LIFE THREATENING ILLness caused almost exclusively by Haemophilus influenzae type b. The majority of cases are in children younger than five years of age, with a peak incidence at age two to four years (1-17). Respiratory distress with impending acute ob-

Winnipeg Children's Hospital, Department of Pediatrics and Child Health, University of Manitoba. Winnipeg. Manitoba: Montreal Children's Hospital, Department of Pediatrics, McGill University, Montreal, Quebec; Izaac Walton Killam Hospital for Children, Department of Pediatrics, Dalhousie University. Halifax, Nova Scotia: Children's Hospital of Eastern Ontario. Department of Pediatrics. University of Ottawa, Ottawa, Ontario; University Hospital, Department of Pediatrics. University of Saskatchewan, Saskatoon, Saskatchewan: and Charles A Janeway Child Health Centre, Department of Pediatrics, Memorial University. St John's, Newfoundland Correspondence and reprints: Dr BJ Law, Department of Medical Microbiology. University of Manitoba, Room 530, 730 William Avenue, Winnipeg, Manitoba R3E OW3. Telephone (204) 788-6629

Received for publication February 13, 1990. Accepted April 3. 1990 struction is the most notable clinical presentation, but evidence of systemic infection with fever and leukocytosis is also present in the majority of cases. Although securing an airway is essential in the immediate management of epiglottitis, the use of parenteral antibiotics is also a mainstay of therapy since bacteremia is documented in 60 to $90 \%$ of cases.

The appropriate duration of total as well as parenteral antibiotic therapy for epiglottitis has not been addressed in controlled randomized prospective studies. A search of the literature revealed a variety of suggested therapeutic regimens providing a total antibiotic course of seven to 10 days $(5,8,12,13)$. Specific recommendations for duration of intravenous therapy varied from $24 \mathrm{~h}$ after either admission (18) or extubation (19), to seven days (20-22). Data supporting these approaches were not found. Prior to the development of a prospective therapeutic trial, a retrospective study in six different Canadian centres was con- 


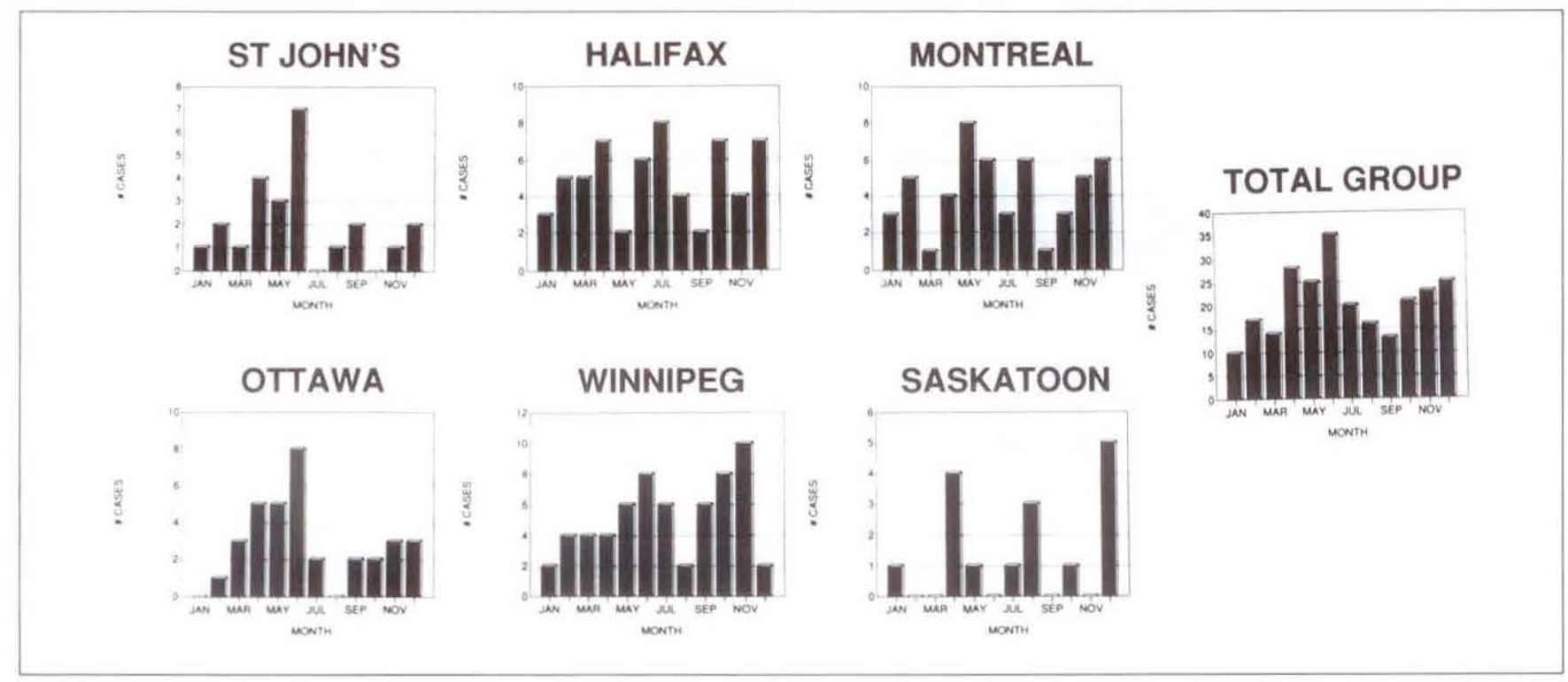

Figure 1) Centre-specific monthly distribution of cases determined using years for which the entire calendar year was available: St John's 1984-87: Halifax 1980-86; Montreal 1982-85; Ottawa 1984-88: Winnipeg 1981-85; and Saskatoon 1982-85

ducted to relate pattern of antimicrobial therapy to hospital course and outcome. Aside from the unique focus on antimicrobial therapy, this review represents the first large series of children with epiglottitis from diverse areas of Canada in terms of location, population density and urbanization.

\section{PATIENTS AND METHODS}

The hospital records of children with a discharge diagnosis of epiglottitis were reviewed in the following centres for the years as specified: Charles A Janeway Child Health Centre (166 beds; referral base 500,000) in St John's. Newfoundland (September 1983 to June 1988); Izaac Walton Killam Hospital for Children (233 beds; referral base 800.000) in Halifax. Nova Scotia (January 1980 December 1986): Montreal Children's Hospital $(214$ beds: referral base $3,000,000$, shared with one other children's hospital) in Montreal, Quebec (April 1981 March 1986): Children's Hospital of Eastern Ontario (230 beds; referral base 1,000,000) in Ottawa, Ontario (May 1982 to June 1986): Winnipeg Children's Hospital (150 beds; referral base 1,200,000) in Winnipeg, Manitoba (February 1980 October 1986); and University Hospital (96 pediatric beds in a general hospital; referral base 500,000) in Saskatoon. Saskatchewan (July 1977 to September 1986).

Data were extracted from each record using a standardized reporting form which included demographic data, presenting symptoms includ- ing initial management prior to arrival at the admitting hospital, management and course while in hospital, laboratory investigations, subsequent management and follow-up after discharge from hospital.

Definite epiglottitis was defined as a characteristic clinical presentation with direct visual evidence of acute inflammation of the epiglottis and/or a positive blood culture for $H$ influenzae type b. Probable epiglottitis was defined as typical clinical symptoms with evidence of an enlarged epiglottis or aryepiglottis on radiograph of the lateral neck in the absence of both direct visualization of the epiglottis and a positive blood culture. Supraglottitis was defined as a typical clinical syndrome in which, on direct visual inspection, the epiglottis was normal but the aryepiglottis and surrounding area were inflamed and edematous, irrespective of culture results. In order to determine duration of antibiotics given in hospital by intravenous as well as oral routes, the actual number of doses of each antibiotic was counted and indicated on the data form along with the dosing interval. The duration for each drug by a specific route was defined as the number of doses multiplied by one twenty-fourth of the dosing interval. The duration of antibiotics following discharge was estimated from the interval specified on discharge orders.

Standard statistical methods were used to analyze differences between centres with respect to presentation of illness, results of laboratory investigations and management in hospital. 


\section{RESULTS}

Patient population/demographic variables: A total of 311 charts were reviewed: 31 from St John's, 61 from Halifax, 66 from Montreal, 47 from Ottawa, 79 from Winnipeg and 27 from Saskatoon. Six cases were eliminated on the basis of age (16 years or older) or diagnosis: three adults from Saskatoon (the only centre which admitted adults), and three cases in which the diagnosis of epiglottitis was not confirmed on chart review. These included one case with croup (Halifax), one with retropharyngeal abscess (Winnipeg) and one with suspected epiglottitis who was hospitalized for less than $24 \mathrm{~h}$ and in whom chest and lateral neck radiographs were normal and blood cultures negative (Saskatoon). Of the remaining 305 cases. 291 were defined as definite epiglottitis, six as probable epiglottitis and eight as supraglottitis.

The mean \pm standard deviation for patient age was $44.8 \pm 26.6$ months with a range of four to 176 months. The peak age of occurrence was from 25 to 48 months ( $48 \%$ of cases). The age distribution did not vary significantly between centres. The proportion of cases potentially preventable by administration of $H$ influenzae type b conjugate vaccine at 18 months of age was $92 \%$ overall with a range of 81 to $100 \%$ between centres.

The male/female ratio was 1.39 . There was an excess of males in all centres except Ottawa where the ratio was 0.88 . Information concerning race was available for $59 \%$ of cases. These were pre-

TABLE 1

Prevalence of presenting symptoms in Canadian children with epiglottitis

\begin{tabular}{|c|c|c|c|c|}
\hline & \multicolumn{2}{|c|}{ Total group } & \multirow{2}{*}{$\begin{array}{c}\text { Low } \\
\text { estimate } \\
\text { range among } \\
\text { centres }\end{array}$} & \multirow{2}{*}{$\begin{array}{c}\text { Previously } \\
\text { reported } \\
\text { range } \\
(1-12)\end{array}$} \\
\hline & $\begin{array}{c}\text { High } \\
\text { estimate }\end{array}$ & $\begin{array}{c}\text { Low } \\
\text { estimate }\end{array}$ & & \\
\hline Fever & $93 \%$ & $89 \%$ & $89-93 \%(5)^{*}$ & $60-100 \%$ \\
\hline Dyspnea & $87 \%$ & $83 \%$ & $79-87 \%(5)$ & $15-100 \%$ \\
\hline Sore throat & $81 \%$ & $69 \%$ & $-(0)$ & $20-82 \%$ \\
\hline Odynophagia & $87 \%$ & $56 \%$ & $76-77 \%(2)$ & $26-90 \%$ \\
\hline Drool & $58 \%$ & $53 \%$ & $44-60 \%(4)$ & $2-91 \%$ \\
\hline Stridor & $55 \%$ & $48 \%$ & $40-64 \%(2)$ & $20-93 \%$ \\
\hline URI & $42 \%$ & $39 \%$ & $31-55 \%(5)$ & $22-26 \%$ \\
\hline Hoarseness & $38 \%$ & $30 \%$ & $15 \%(1)$ & $4-38 \%$ \\
\hline Emesis & $28 \%$ & $25 \%$ & $15-35 \%(5)$ & $25 \%$ \\
\hline Cough & $27 \%$ & $24 \%$ & $14-35 \%(4)$ & $0-40 \%$ \\
\hline
\end{tabular}

High estimate calculation included in the denominator only patients for whom absence or presence of symptoms was specified on the report form: low estimate calculation used the total group $(n=305)$ as the denominator. The range of centre-specific symptom prevalence is given for centres in which presence or absence of symptoms was specified for at least $90 \%$ of patients. " Number of centres qualifying. URI Upper respiratory infection dominantly Caucasian $(94 \%)$ with the remainder being Black and Oriental. There were no Amerindian cases identified. The overall and centrespecific distributions of cases by month are shown in Figure 1.

Presentation of illness: The prevalence of presenting symptoms is shown in Table 1 . The median duration of illness (90th percentile; range) prior to admission was $1.0(5.0 ; 0.1$ to 48$)$ days. Corresponding values for individual symptoms were: upper respiratory infection $3.0(7 ; 0$ to 21$)$; cough 1.0 (7; 0.1 to 3.0$)$; fever $0.6(2.0 ; 0.1$ to 7.0$)$; sore throat 0.5 (2.0; 0.1 to 14$)$; odynophagia 0.5 (1.0; 0.1 to 4 ); hoarse voice 0.3 (1.0; 0.1 to 3 ); emesis 0.3 (1.0; 0.1 to 2$)$; drooling $0.2(0.8 ; 0.1$ to 2$)$; dyspnea $0.2(0.5 ; 0.1$ to 5.0$)$; and stridor $0.2(0.5$; 0.1 to 4$)$. Prior to admission, 113 children (40\%) were seen by a physician. A diagnosis of epiglottitis was made for $34 \%$ of the children seen. One or more doses of antibiotics were received by $23 \%$ of the children prior to arrival at the admitting hospital. There were no significant differences between the six centres for any of these parameters (ANOVA: $P>0.1$ ).

Therapy for epiglottitis was initiated at a location other than the admitting hospital for 118 (39\%) children, including another hospital for 109, a physician's office for six and 'not stated' in three. Among the 109 patients first seen at a referring hospital, airway management prior to transfer included none in 39\%, intubation in 51\% and tracheotomy in $5 \% ; 12 \%$ were reported to have had respiratory arrest. The proportion of referred patients varied significantly between centres: Montreal 18\%; Ottawa 38\%: St John's 39\%; Halifax 40\%; Winnipeg 50\%; and Saskatoon 56\% $\left(\chi^{2}=35 ; \mathrm{P}<0.0001\right)$.

The initial assessment was performed at the admitting hospital for $187(61 \%)$ patients. Respiratory arrest occurred in $2 \%$. In the majority of instances, the patients were transferred immediately to the intensive care unit or operating room, and thus there was little information as to status when seen in the emergency department. Fever (temperature greater than $38 \mathrm{oC}$ ) was documented in $60 \%$,

Airway management in hospital: Overall, 93\% of cases were intubated, $3 \%$ had a tracheostomy and $4 \%$ were observed. None of the patients who were initially observed required intubation. The majority of intubations were done in the operating room. primarily by an anesthetist. Accidental extubation occurred in nine cases (3\%), seven of which were re-intubated. No complications secondary to accidental extubation were reported.

The centre-specific time to extubation is shown in Figure 2. The mean duration of intubation was 
$56 \pm 23 \mathrm{~h}$ (range 17 to 188 ) for the entire group. There was significant variation between centres for this parameter with centre means \pm SD as follows: Ottawa $46 \pm 15$, Halifax $48 \pm 20$; Winnipeg

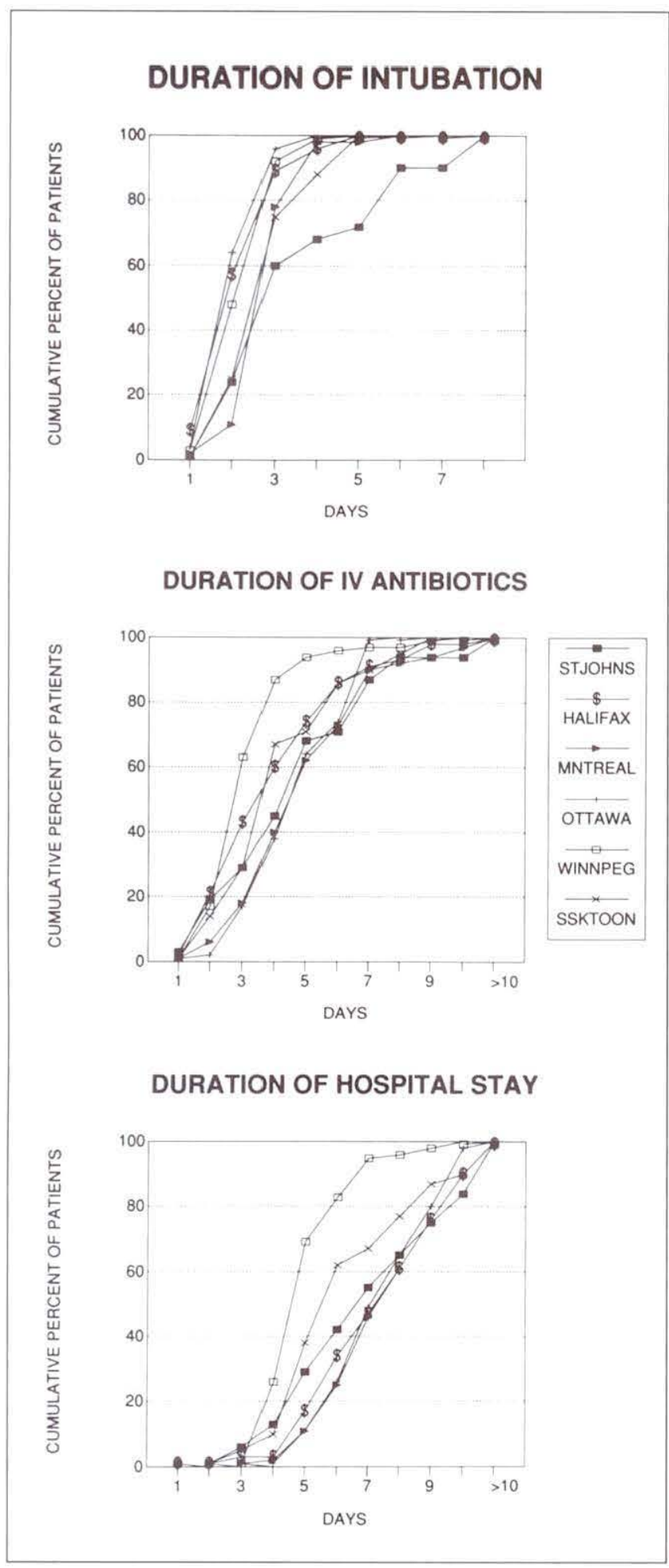

Figure 2) Centre-specific duration of intubation (top): antibiotics (middle); and hospital stay (bottom) for 305 children with acute epiglottitis. The significant differences between centres for each parameter are discussed in the text
$52 \pm 16$; Saskatoon $61 \pm 23$; Montreal $62 \pm 16$; and St John's $81 \pm 44$ (ANOVA: $\mathrm{P}<0.0001$ ).

Post extubation complications included: stridor in $21 \%$, atelectasis in $4 \%$, pneumonia in $2 \%$ and wheeze in $1 \%$. Post extubation stridor was reported more frequently in children who were reported to have had a two stage oral then nasal intubation $(30 \%$ of 122$)$ versus children with a single orotracheal ( $9 \%$ of 11$)$ or nasotracheal $(12 \%$ of 115$)$ intubation procedure $\left(\chi^{2}=12.75 ; \mathrm{P}=0.002\right)$. Stridor was mild, however, and responded in all cases to medical management consisting of mist therapy and/or racemic adrenaline. Other complications included one vallecular cyst, one laryngeal ulcer and one bruised larynx.

Investigations in hospital: A white blood cell (WBC) count was determined shortly after admission for $280(92 \%)$ patients. Leukocytosis defined as total WBC greater than $10.0 \times 10^{9} / \mathrm{L}$ was present in $89 \%$ of patients. The elevation in total WBC was moderate to marked in most patients: 15 to $20 \times 10^{9} / \mathrm{L}$ in $27 \% ; 21$ to 25 in $22 \% ; 25$ to 30 in $13 \%$; and more than 30 in $7 \%$. The absolute band count was less than $0.5 \times 10^{9} / \mathrm{L}$ in $15 \%, 0.5$ to less than 1.0 in $9 \%, 1.0$ to 5.0 in $53 \%$ and more than 5.0 in $23 \%$. A platelet count was recorded for $71 \%$ of cases and was normal in 99\%. The mean hemoglobin was $120 \pm 10 \mathrm{~g} / \mathrm{L}$. A value of less than 110 was noted for $15 \%$.

Blood cultures were obtained for 292 (96\%) patients and were positive in $82 \%$. All but three of the 241 isolates were $H$ influenzae $(99 \%)$. The exceptions (group B beta-hemolytic streptococcus; Haemophilus parainfluenzae; peptococcus) all occurred as single blood isolates in three Montreal patients. All but eight of the $H$ influenzae isolates were typed, and of these, all were capsular type b. Receipt of antibiotics prior to admission significantly decreased the likelihood that a blood culture would be positive. Only 56\% (40 of 71) of patients given antibiotics prior to admission had positive blood cultures versus 91\% (200 of 220) of those who had not received prior treatment $\left(\chi^{2}=42.0\right.$; $\left.\mathrm{P}<0.0001\right)$. Urine for rapid antigen detection was obtained from only 28 patients and was positive for $H$ influenzae type b in 14. Test results suggested an etiology of $H$ influenzae in four patients with negative blood cultures. Among $H$ influenzae type b isolates, $24 \%$ were resistant to ampicillin. There were no significant centre-specific differences in the prevalence of ampicillin resistance: Halifax 20\% (nine of 46), St John's $36 \%$ (eight of 22), Montreal 24\% (14 of 55), Ottawa $28 \%$ (10 of 36), Saskatoon $20 \%$ (one of five), and Winnipeg 23\% (16 of 69 ).

Cultures of the epiglottis were obtained in 207 patients. $H$ influenzae was recovered from $62 \%, H$ 
parainfluenzae from $8 \%$, Staphylococcus aureus from $11 \%$, group A beta-hemolytic streptococcus from $3 \%$, group B beta-hemolytic streptococcus from $0.5 \%$ and mixed normal pharyngeal flora from $14 \%$. There was no growth in $15 \%$. Beta-lactamase activity was determined for paired blood and epiglottis isolates in 58 of the 118 cases in which $H$ influenzae was isolated from both sites. Concordant results were obtained for 57 of 58 $(98 \%)$. In one case, the blood isolate was beta-lactamase positive, whereas the epiglottis isolate was beta-lactamase negative. Most epiglottis H influenzae isolates were not serotyped, and thus correlation with blood culture results could not be assessed.

Radiographs of the chest were obtained for 243 children $(80 \%)$ with reported results as follows: normal $52 \%$, consolidation $29 \%$, atelectasis $14 \%$, pneumothorax $1 \%$, pulmonary edema $1 \%$ and other abnormalities $4 \%$.

Radiographs of the lateral neck were obtained for 243 patients (80\%). Abnormalities were noted in $99 \%$ with findings typical of epiglottitis in $91 \%$. aryepiglottic swelling only in $4 \%$, subglottic swelling in $1 \%$ and uninterpretable changes in 3\%.

Antibiotic therapy: All but one patient received at least one dose of antibiotic intravenously while in hospital. Empiric therapy on admission consisted of ampicillin and chloramphenicol in $81 \%$, chloramphenicol alone in 10\%, ampicillin alone in $1 \%$, cefuroxime in $5 \%$, cefamandole in $1 \%$ and miscellaneous in 1\% (one ampicillin/cefotaxime, one ampicillin/cloxacillin, and one chloramphenicol/cloxacillin). A single patient was started on oral amoxicillin. This patient was observed in hospital without intubation and had no blood culture taken. The diagnosis was based on a lateral neck radiograph which showed swollen aryepiglottic folds and a slightly swollen epiglottis.

Eight patients were excluded from the analysis of duration of antibiotic therapy: four who died early in the hospital course and four with $\mathrm{H}$ influenzae bacteremia in whom the presence of meningitis (one patient) or septic arthritis (three patients) determined the total duration of therapy.

For the group as a whole, the mean $\pm \mathrm{SD}$ duration of intravenous antibiotics was $4.8 \pm 2.2$ days (range 1.3 to 18.5 days); hospital-monitored antibiotics (intravenous + oral) was $6.0 \pm 2.3$ days (range two to 19.5 days); and of estimated total antibiotics (hospital + planned discharge medications) was $9.9 \pm 2.8$ days (range two to 19.8 days). Oral antibiotics were started in hospital for $69 \%$ of patients; these included amoxicillin in $62 \%$, chloramphenicol in $27 \%$, ampicillin in $7 \%$, cefaclor in $3 \%$ and an unspecified antistaphylococcal penicillin in $1 \%$. Orders for oral antibiotics following discharge were written for $73 \%$ of patients. The duration was specified for 186 of 209 patients $(89 \%)$ and averaged $5.2 \pm 2.8$ days. The drugs used were similar to those started in hospital.

Centre variation in duration of antibiotic therapy was analyzed for hospital-monitored therapy only, as compliance with discharge medication could not be determined. The duration (mean \pm SD) of intravenous antibiotics was significantly shorter in Winnipeg (3.8 \pm 1.4 days) than in Montreal (5.7 \pm 2.2 days), Ottawa (5.0 \pm 1.5 days) and St John's $(5.3 \pm 2.7$ days $)(\mathrm{P}<0.05$ by Tukey's studentized range test). The durations in Halifax ( $4.8 \pm 2.6$ days) and Saskatoon ( $4.7 \pm 2.0$ days) were not significantly different from the other centres. The centre-specific cumulative duration of intravenous antibiotics is shown in Figure 2. The mean duration of intravenous plus oral antibiotics administered in hospital was also significantly shorter for patients from Winnipeg ( $5.4 \pm 1.5$ days) compared with all other centres $(6.6 \pm 2.9 ; 6.7 \pm$ $2.8 ; 6.6 \pm 2.1$; and $6.8 \pm 1.4$ days) except Saskatoon ( $5.7 \pm 1.8$ days). The presence or absence of documented bacteremia had no significant effect on the duration of either intravenous ( $4.9 \pm$ 2.2 and $4.2 \pm 1.8$ days, respectively) or total hospital monitored antibiotics $(6.1 \pm 2.3$ and $5.7 \pm 2.2$, respectively).

Hospital course: During hospitalization, fever (temperature greater than $38^{\circ} \mathrm{C}$ irrespective of site of measurement) was documented in $88 \%$ of patients. The maximum recorded temperature in hospital occurred on the first day for $84 \%$ of patients, the second day for $10 \%$ and the third day or later for $6 \%$. The mean duration of fever (defined as first hospital day afebrile minus one) was $1.9 \pm$ 1.3 days overall.

The discharge diagnosis indicated an extraepiglottic focus of infection in 46 patients (15\%) including 25 pneumonia; 13 otitis media; three septic arthritis (one with associated osteomyelitis); three cervical adenitis; one pharyngitis; one mediastinitis; one submandibular cellulitis; and one meningitis. Clinical data for the children with septic arthritis and meningitis are summarized in Table 2. All four children had bacteremia with beta-lactamase negative $H$ influenzae, and none had received antibiotics prior to admission. The duration of fever was significantly longer (ANOVA: $\mathrm{P}<0.0001)$ in the children with a second site of infection ( $2.9 \pm 1.7$ days) than in children with epiglottitis only ( $1.9 \pm 1.1$ days). The maximum recorded temperature in hospital equalled or exceeded $40^{\circ} \mathrm{C}$ in $33 \%$ of children with an extraepiglottic site of infection versus $13 \%$ of children with epiglottitis only $\left(\chi^{2}\right.$ test, $\left.\mathrm{P}=0.002\right)$. The admitting leukocyte count was not helpful in identifying children with a second focus of infection. 
TABLE 2

Clinical data for four children with a second invasive focus of infection accompanying epiglottitis

\begin{tabular}{|c|c|c|c|c|c|c|c|c|c|}
\hline $\begin{array}{c}\text { Age } \\
\text { (years) }\end{array}$ & Sex & $\begin{array}{l}\text { Days ill } \\
\text { PTA }\end{array}$ & WBC & $\begin{array}{c}\text { Maximum } \\
\text { temperature }\left({ }^{\circ} \mathrm{C}\right)\end{array}$ & $\begin{array}{l}\text { First } \\
\text { afebrile day }\end{array}$ & $\begin{array}{l}\text { Recurrent } \\
\text { fever (day) }\end{array}$ & Extraepiglottic site & $\begin{array}{l}\text { Hospital } \\
\text { day onset }\end{array}$ & $\begin{array}{l}\text { Diagnosis } \\
\text { confirmed by }\end{array}$ \\
\hline 2.0 & $M$ & 2 & 18.9 & 40.3 & 4 & Yes (7) & Right knee & $?$ & Bone scan \\
\hline 3.2 & $M$ & 1 & 17.7 & 40.3 & 3 & No & Left elbow & 6 & Not stated \\
\hline 6.3 & $F$ & 7 & 16.9 & 39.8 & 6 & No & $\begin{array}{l}\text { Right ankle and dis- } \\
\text { tal tibia }\end{array}$ & 5 & Gallium scan \\
\hline 3.2 & $\mathrm{~F}$ & 0.5 & 23.0 & 40.5 & 4 & Yes (6) & Meninges & 4 & CSF culture \\
\hline
\end{tabular}

PTA Prior to admission. "Lumbar puncture done on admission because of lethargy. Cerebrospinal fluid (CSF) had zero cells and was culture negative. Repeat CSF on the fourth hospital day had 682 cells ( $98 \%$ polymorphonuclear leukocytes) and grew Haemophilus influenzae type b

Four children died, for a case fatality rate of $1.3 \%$. In all four cases, cardiorespiratory arrest occurred prior to transfer and the triage site was $120 \mathrm{~km}$ or more from the admitting hospital. Respiratory arrest occurred in two of the children at time of presentation and, despite resuscitation. death occurred within 5 and $18 \mathrm{~h}$ of admission to hospital. Post mortem information was not available. One child had a large tension pneumothorax and subcutaneous emphysema of the neck. The other two had been managed as croup at a referring hospital for several hours prior to acute deterioration and transfer. Both of these children died two days after transfer. Post mortem examination confirmed the diagnosis of epiglottitis and provided evidence of hypoxic encephalopathy and pulmonary edema.

Complications noted among survivors included one bleeding stress ulcer, one febrile seizure, and one case of cerebral irritability related to a postoperative medication.

\section{DISCUSSION}

Despite the geographic separation and regional differences between the six centres included in this study, patient demographics, clinical presentation, hospital course and outcome of acute epiglottitis, admitting laboratory data including complete blood count and radiography, and etiology including prevalence of ampicillin-resistant $H$ influenzae type b were remarkably similar. A recent review from Toronto's Hospital for Sick Children presented a similar clinical picture (12). These results were not unexpected given the uniformity of presentation and course reported in review articles from several different countries, including the United States $(2-4,9-11,17,19)$. England (18), Australia (14), New Zealand (7). Switzerland (8) and Sweden (15).

The primary focus of this review was to document the duration of intravenous antibiotic therapy currently practised in several Canadian centres and to determine whether or not a very short course of intravenous antibiotic was associated with recurrent infection. Significant centre- related differences in management were apparent, with the shortest course of both intravenous and hospital-monitored antibiotic therapy employed in Winnipeg. Among 78 patients treated there, 63 and $69 \%$, respectively received intravenous antibiotics for at most three days and stayed in hospital for no more than five days. The corresponding figures for the entire series were 36 and $31 \%$. respectively.

Recurrences of epiglottitis or other sites of infection were not documented for any of the children in this series. Minor infections treated at a physician's office or more serious infections managed in a different hospital could, however, have been missed. Return visits after hospitalization for epiglottitis were recorded for $46 \%$ of the children reviewed. Problems potentially related to the acute illness were noted for only $2 \%$ of this group, and none of these were for recurrence of infection. A review of the literature revealed only one instance of readmission for recurrent epiglottitis. This was one of 349 cases reported from Australia (14). The recurrence occurred in a child who had been in hospital for $24 \mathrm{~h}$ only.

The optimal duration of parenteral and hospital-monitored antibiotic therapy is unknown. Factors which may contribute to complications include the presence and magnitude of the bacteremia, as well as the presence or absence of a second focus of infection. Nearly all of the children reviewed had blood cultures drawn, and $82 \%$ had documented bacteremia. For children who received no oral antibiotics prior to admission, the prevalence of bacteremia was even higher at $91 \%$. This is in agreement with the currently held view that most cases of clinical epiglottitis in children are due to $H$ influenzae type $\mathrm{b}$. and that bacteremia is probably universally present at some time during the course of the illness. The magnitude of bacteremia in epiglottitis is 10 to 1000 colony forming units per mL, which is 1 to $10 \%$ of that found in $H$ influenzae type b meningitis (23). This may explain the reported range of 50 to $90 \%$ for positive blood cultures in epiglottitis, as well as the present observation of a significantly lower 
prevalence of documented bacteremia (56\%) among the 71 children in this series who received oral antibiotics prior to admission. Furthermore, the rarity of reported concurrent sites of invasive infection - including meningitis, septic arthritis and cellulitis $(24,25)$ - support the concept that bacteremia is not a major part of the pathogenesis of epiglottitis but probably develops secondarily to the marked degree of inflammation of the epiglottis and surrounding structures in conjunction with mucosal colonization. This line of reasoning would support a relatively short duration not only of parenteral but also of total antibiotic therapy. The majority of the $233(76 \%)$ children in this series who had no extraepiglottic sites of infection identified during the hospital course had a very rapid defervescence over two to four days. In this subgroup, one or two daily doses of an antibiotic with a long half-life, such as ceftriaxone, given for the duration of endotracheal tube placement and followed by a few days of oral antibiotics, may be sufficient.

For children with a second focus of infection. the length and type of antibiotic therapy would depend upon the specific site of extraepiglottic infection. As noted above, most reviews of epiglottitis stress the low frequency of secondary sites of infection. The most extensive review to date was done by Molteni (24) who reported a $25 \%$ incidence of both pneumonia and cervical lymphadenitis, $8 \%$ pharyngitis and $5 \%$ otitis media among 72 patients from his own institution. The reported incidence of concurrent pneumonia ranges from 1 to $50 \%$. Unfortunately, the validity of a clinical diagnosis of pneumonia versus atelectasis is impossible to establish in a retrospective review. For the 305 patients in this review, a diagnosis of pneumonia was based on a stated discharge diagnosis as well as $\mathrm{x}$-ray findings, and thus the observed rate of $8 \%$ may have underestimated the true incidence.

There have been at least one dozen reported cases of concurrent epiglottitis and meningitis. To the authors' knowledge, septic arthritis and osteomyelitis have not previously been reported as accompanying epiglottitis. In one of the three children in the present series, the diagnosis was only suspected after six days in hospital, at which time the patient was afebrile; the hospital course had been uncomplicated. The other two children with septic arthritis and the one with meningitis had unusual hospital courses with fever which was either slow to resolve or recurrent after an afebrile period. These cases, although infrequent, must be considered in determining the optimal duration and route of antibiotic therapy and the period of observation in hospital. Investigation to rule out a second focus of infection should be carefully considered in children with fever exceeding $40^{\circ} \mathrm{C}$ or persisting four days or more after admission to hospital.

The data gathered in this review raise questions about issues other than rate and duration of antibiotic therapy. As shown in Table 1, the reported prevalence of presenting symptoms for epiglottitis varies over a wide range even for the classically recognized tetrad of dyspnea, drool, dysphagia and altered voice. This finding likely follows from the unreliable nature of retrospective reviews. In most reports, it is impossible to tell whether or not the absence of a given symptom relates to absence of recorded data or absence of a clinical finding. A recent report of four cases of 'atypical' epiglottitis stressed that children may present with a more gradual illness evolving over several days with symptoms, such as cough and hoarse voice, that are more usually associated with croup (26). Although acute onset of respiratory distress occurred in most children in this series, several had been ill for two to five days and $24 \%$ of the group had been seen by a physician prior to presentation at hospital with an illness that was not diagnosed as epiglottitis. Furthermore, one of the four deaths occurred in a child who had a sudden respiratory arrest while being managed as croup at a referring hospital. A prospective study of epiglottitis would give a much clearer picture of the natural history immediately preceding the acute onset of respiratory distress. Nevertheless, the data presented here serves to emphasize that an 'atypical' presentation of epiglottitis evolving over a few days is perhaps more common than previously acknowledged.

Most of the data from this series regarding airway management, including the lack of significant complications associated with intubation, is consistent with previous reports. The increased incidence of post extubation stridor associated with a two stage orotracheal to nasotracheal intubation was unexpected. The retrospective nature of the study with lack of an objective definition of stridor makes any conclusion to be drawn from this finding tenuous. Nevertheless, given the degree of inflammation and edema which accompanies acute epiglottitis, the possibility of greater damage with a two stage intubation procedure deserves further study.

The wide variety of bacterial isolates recovered from culture swabs of the epiglottis as well as the one instance in which an ampicillin-sensitive $H$ influenzae was isolated from the epiglottis whereas an ampicillin-resistant $H$ influenzae was recovered from blood, underscores the lack of reliability of epiglottis cultures for determining 
antibiotic choice. In the absence of a blood isolate, management with a beta-lactamase stable antibiotic should be continued, irrespective of the sensitivity of isolates from the epiglottis.

In conclusion, this review provides baseline data on the clinical presentation and course of epiglottitis in children from several regions of Canada. As with other retrospective reviews of epiglottitis. it raises many questions. Despite a multitude of reviews of epiglottitis over the past four decades involving nearly 4500 children, there remain many unresolved issues surrounding the pathogenesis, natural history and management of epiglottitis. These could potentially be addressed in a multicentre prospective therapeutic trial com-

\section{REFERENCES}

1. Dajani AS, Asmar BI, Thirumoorthi MC. Systemic Haemophilus influenzae disease: An overview. J Pediatr 1979;94:355-64.

2. Bass JW, Steele RW, Wiebe RA. Acute epiglottitis. A surgical emergency. JAMA 1974:229:671-5.

3. Johnson GK, Sullivan JL, Bishop LA. Acute epiglottitis. Review of 55 cases and suggested protocol. Arch Otolaryngol 1974;100:333-7.

4. Battaglia JD, Lockhart $\mathrm{CH}$. Management of acute epiglottitis by nasotracheal intubation. Am J Dis Child 1975; 129:334-6.

5. Lacroix J, Blanc VF. Weber M, Perreault G. Etude de 100 cas consêcutifs d'épiglottite aiguë. Union Med Can 1982:111:774-9.

6. Willis RJ, Rowland TW. The early management of acute epiglottitis: A survey of current practice. J Emerg Med 1984:2:13-6.

7. McGregor AR, Dawson KP. Abbott GD. Acute epiglottitis in childhood, Christchurch 1970-84. N Z Med J 1985:98:1011-3.

8. Gerber AC, Pfenninger J. Acute epiglottitis: Management by short duration of intubation and hospitalization. Intensive Care Med 1986:12:407-11.

9. Gonzalez C, Reilly JS, Kenna MA. Thompson AE. Duration of intubation in children with acute epiglottitis. Otolaryngol Head Neck Surg 1986;95:477-81.

10. Vernon DD. Sarnaik AP. Acute epiglottitis in children: A conservative approach to diagnosis and management. Crit Care Med 1986;14:23-5.

11. Kimmons HC Jr, Peterson BM. Management of acute epiglottitis in pediatric patients. Crit Care Med 1986;14:278-9.

12. Sendi K, Crysdale WS. Acute epiglottitis: Decade of change - A 10-year experience with 242 children. J Otolaryngol 1987; 16:196-202.

13. Daum RS, Smith AL. Epiglottitis (supraglottitis). In: Feigin RD, Cherry JD, eds. Textbook of Pediatric Infectious Diseases, 2nd edn. Philadelphia: WB Saunders Company, 1987:224-36.

14. Butt W, Shann F, Walker C, Williams J, Duncan A. Phelan P. Acute epiglottitis: A different approach paring a short course of intravenous antibiotics alone to sequential intravenous-oral combined therapy for seven to 10 days. Of note is that $92 \%$ of cases in this series were potentially preventable with the administration of conjugate $H$ influenzae type $b$ vaccine at 18 months of age. This vaccine was not in use during the period covered by this review. Thus, it is possible that the incidence of epiglottitis in Canada will be substantially reduced by the introduction of this vaccine, making a large therapeutic study difficult. Perhaps a more important issue than optimal duration of therapy is the need for early recognition and appropriate emergency management in the hopes of preventing deaths secondary to epiglottitis.

to management. Crit Care Med 1988; 16:43-7.

15. Claesson B, Trollfors B, Ekstrom-Jodal B, et al. Incidence and prognosis of acute epiglottitis in children in a Swedish region. Pediatr Infect Dis 1984;3:534-8.

16. Bass JW, Fajardo JE, Brien JR, Cook BA, Wiswell TE. Sudden death due to acute epiglottitis. Pediatr Infect Dis 1985:4:447-9.

17. MayoSmith MF, Birsch PJ. Wodzinski SF, Schiffman FJ. Acute epiglottitis in adults. An eight-year experience in the state of Rhode Island. N Engl J Med 1986;314:1133-9.

18. Drake-Lee AB, Broughton SJ, Grace A. Children with epiglottitis. Br J Clin Pract 1984;38:218-42.

19. Diaz JR, Lockhart $\mathrm{CH}$, Early diagnosis and airway management of acute epiglottitis in children. South Med J 1982;75:399-403.

20. Schloss MD, Gold JA, Rosales JK, Baxter JD. Acute epiglottitis: Current management. Laryngoscope 1983:93:489-93.

21. Blanc VF, Weber ML, Leduc C, Laberge R, Desjardins R, Perreault G. Acute epiglottitis in children: Management of 27 consecutive cases with nasotracheal intubation, with special emphasis on anaesthetic considerations. Can Anaesth Soc J 1977:24:1-11.

22. Bottenfield GW, Arcinue EL, Sarnaik A, Jewell MR. Diagnosis and management of acute epiglottitis Report of 90 consecutive cases. Laryngoscope 1980:90:822-5.

23. La Scolea LJ Jr, Rosales SV, Welliver RC, Ogra PL. Mechanisms underlying the development of meningitis or epiglottitis in children after Haemophilus influenzae type b bacteremia. J Infect Dis 1985;151:1162-5.

24. Molteni RA. Epiglottitis: Incidence of extraepiglottic infection: Report of 72 cases and review of the literature. Pediatrics 1976;58:526-31.

25. Friedman EM, Damion J, Healy GB, McGill TJI. Supraglottitis and concurrent haemophilus meningitis. Ann Otol Rhinol Laryngol 1985;94:470-2.

26. Schuh S, Huang A, Fallis JC. Atypical epiglottitis. Ann Emerg Med 1988:17:168-70. 


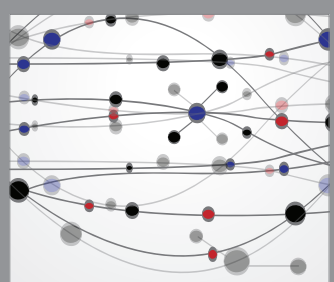

The Scientific World Journal
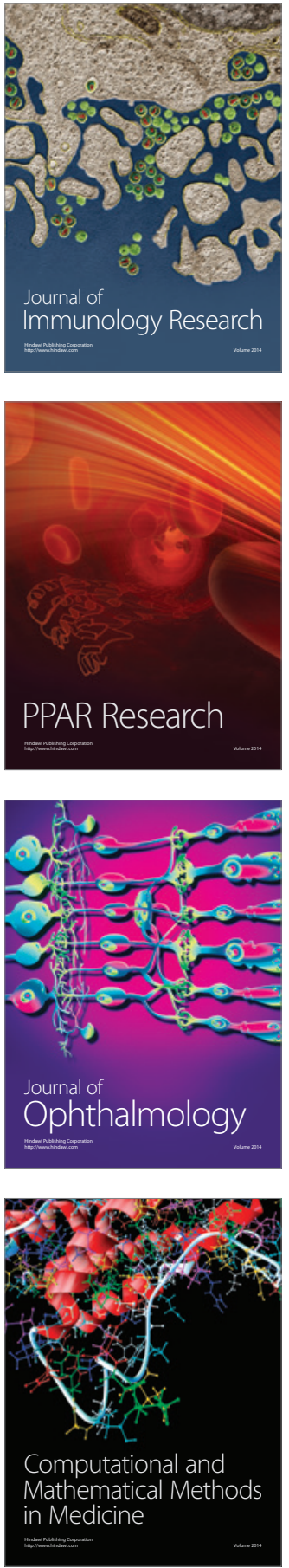

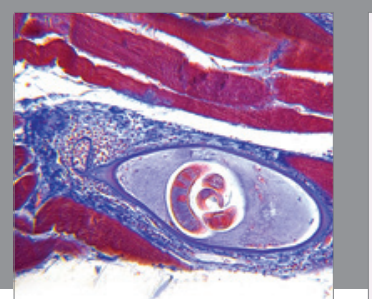

Gastroenterology Research and Practice

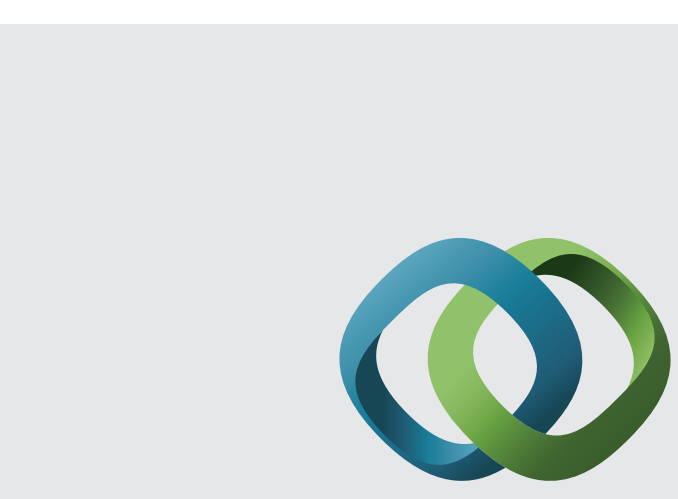

\section{Hindawi}

Submit your manuscripts at

http://www.hindawi.com
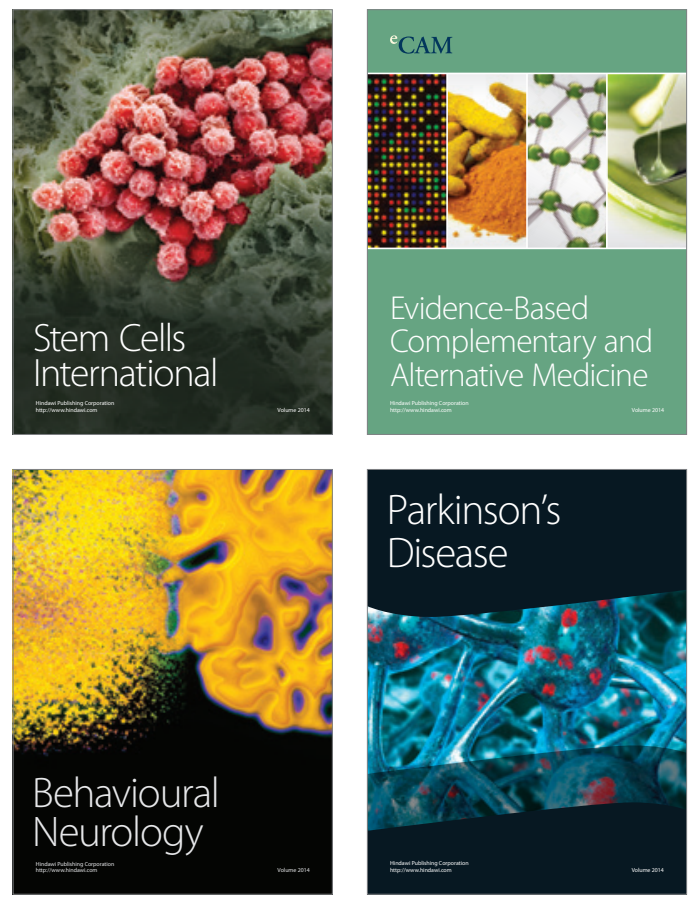
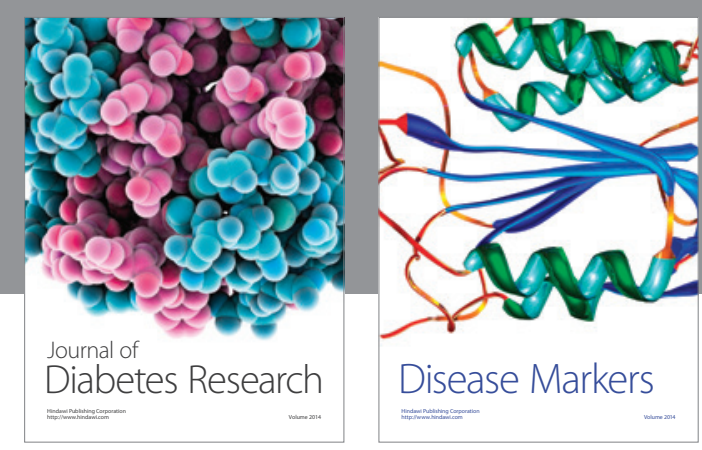

Disease Markers
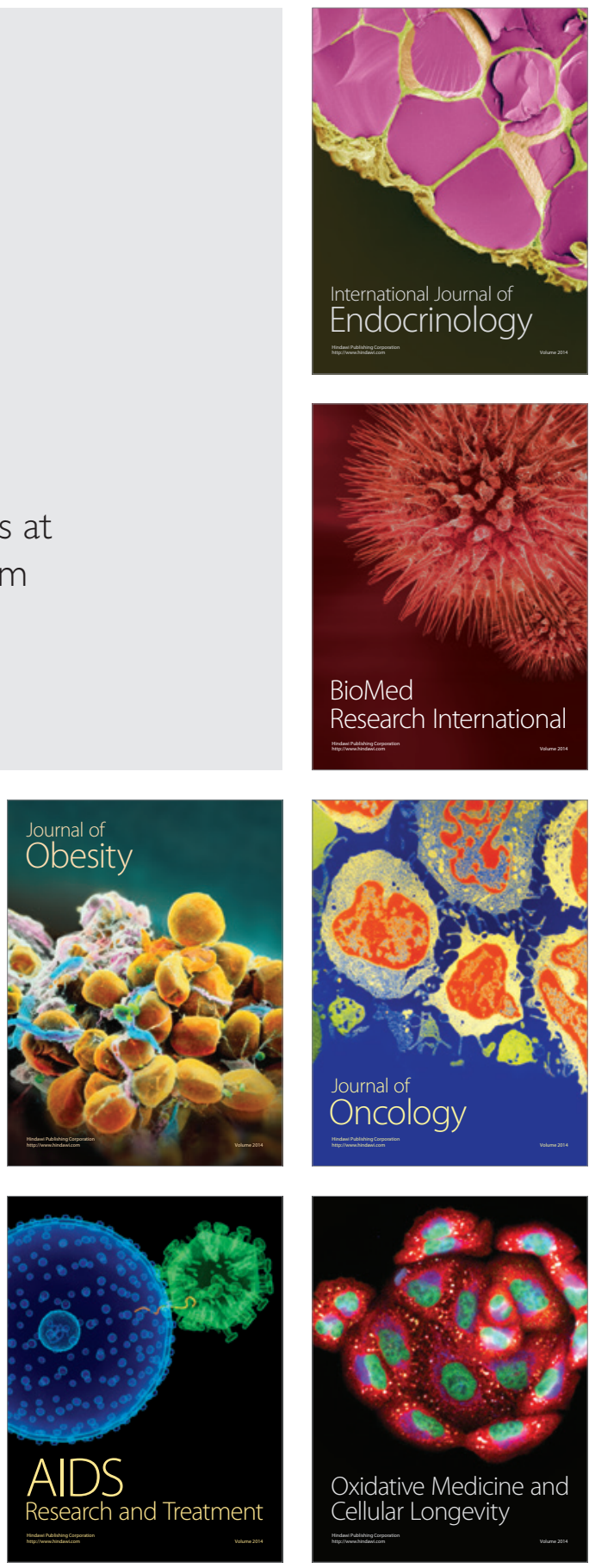